\title{
Teaching of removable prosthodontics in the UK
}

At the recent British Society for the Study of Prosthetic Dentistry (BSSPD) conference, representatives discussed the all important challenges encountered in the delivery of undergraduate training in removable prosthodontics. The group set out to answer the following questions:

- What are the current expectations of undergraduates in relation to their output within removable prosthodontics?

- What are the constraints on providing undergraduates with an adequate quality and quantity of clinical experience?

While the General Dental Council states that on graduation, students should be competent at providing partial and complete dentures, the group questioned what level of 'competency' could be realistically achieved within the five undergraduate years. Although there was no doubt that basic skills could be acquired, it was felt that only with clinical practice and exposure could a sufficient degree of competence be reached. Depending on the school, most UK qualifying students manage between four and 13 patients requiring removable prostheses and on average three of the cases (range 1-8) are edentulous patients. Although several of the schools also include competence assessment in various aspects of removable prosthodontics, there was doubt as to how much confidence there could be in this in the current climate. Certainly, there is a need to ensure rigorous assessment of competence at a time of decreased clinical exposure and this has to be assured wherever possible.

What then are the constraints that limit an undergraduate's experience? Firstly, there are a decreasing number of patients deemed suitable for the provision of removable prostheses by inexperienced students, particularly patients requiring complete dentures who are often referred by experienced practitioners, unable to satisfy their treatment needs. Also, the changing demographics of patients requiring removable prostheses means that whilst the prevalence of edentulism is decreasing, the age of becoming edentulous is increasing. There was a perception among the group that the general difficulty, with respect to patient type and clinical complexity, is also on the increase. Add to this the general reduction in clinical exposure to these patients at dental schools and the challenges become more apparent at both undergraduate and postgraduate levels.

Many of the UK dental schools teach restorative dentistry in 'polyclinics' or outreach centres in order to embrace the concept of total patient care. These clinics are often staffed by skilled and dedicated general dental practitioners and their value in delivering an increased amount of clinical teaching was not underestimated by the group; however, these staff members are mainly generalists and do not claim to have specialist skills in removable prosthodontics. Concern was expressed, therefore, that overall control of the teaching and delivery of removable prosthodontics could be easily lost as the number of specialist clinical academics with suitable skills reduces. The ever increasing burden of fewer teachers providing greater clinical support and teaching was of concern in terms of their continuing effectiveness.

Removable prosthodontics is one area of dentistry that relies heavily on the interaction of members of the dental team with clinician and technician working closely together. The reduction in the number of technician training posts and instructor technicians has resulted in several schools reducing the quota of cases that they expect students to complete, because they would be unable to cope with the amount of technical support required within available resources. At the inception of vocational training (VT) it was intended to provide additional experience and training in all aspects of dentistry. However, with regard to removable prosthetics, many of the group reported - albeit anecdotally- that graduates felt their prosthodontic skills had decreased rather than improved after their VT year.

The group has proposed to conduct a formal and detailed survey of the teaching of removable prosthodontics in UK dental schools and to make recommendations to lessen any negative impact on the provision of prosthodontic services in the future.

The group consisted of the following members: Janice Ellis - Rapporteur (Newcastle), Robert McAndrew - Assistant rapporteur (Cardiff), John Hobkirk (Chair, Eastman), Michael Fenlon (Kings College London), Frank Houston (Dublin), Mike Barsby (Queen Mary), Fraser McCord (Manchester), Tom Clifford (Belfast), Rob Jagger (Bristol), Nicolas Martin (Sheffield), Brendan Scott (Dundee), Damien Walmsley (Birmingham), Mervyn Lyons (Glasgow), Derek Adams (Liverpool), Chris Watson (Leeds) and Finbarr Allen (Cork). j.s.ellis@newcastle.ac.uk

Stephen Hancocks OBE, Editor in Chief doi: $10.1038 /$ sj.bdj.4813910 